STRUCTURAL BIOLOGY

ISSN 2059-7983

\section{PRISM-EM: template interface-based modelling of multi-protein complexes guided by cryo-electron microscopy density maps. Corrigendum}

\author{
Guray Kuzu, ${ }^{a} \neq$ Ozlem Keskin, ${ }^{a, b *}$ Ruth Nussinov ${ }^{c, d}$ and Attila Gursoy ${ }^{e_{*}}$
}

${ }^{a}$ Center for Computational Biology and Bioinformatics and College of Engineering, Koc University, 34450 Istanbul, Turkey, ${ }^{\mathbf{b}}$ Chemical and Biological Engineering, College of Engineering, Koc University, 34450 Istanbul, Turkey, ${ }^{\mathbf{c} C a n c e r}$ and Inflammation Program, Leidos Biomedical Research Inc., National Cancer Institute, Frederick National Laboratory for Cancer Research, Frederick, MD 21702, USA, ${ }^{\mathbf{d}}$ Sackler Institute of Molecular Medicine, Department of Human Genetics and Molecular Medicine, Sackler School of Medicine, Tel Aviv University, Tel Aviv 69978, Israel, and ${ }^{\mathbf{e}}$ Computer Engineering, Koc University, 34450 Istanbul, Turkey. *Correspondence e-mail: okeskin@ku.edu.tr, agursoy@ku.edu.tr

Edited by G. J. Kleywegt, EMBL-EBI, Hinxton, England

₹ Current address: Department of Molecular Biology, Massachusetts General Hospital, Boston, MA 02114, USA.

Keywords: multimolecular protein complexes; modelling protein assemblies; PRISM-EM; three-dimensional electron microscopy; protein structure prediction.

Supporting information: this article has supporting information at journals.iucr.org/d

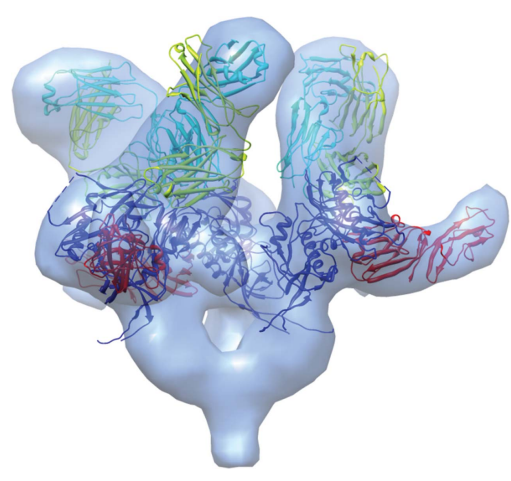

C 2018 International Union of Crystallography
A revised Table 6 and Supporting Information are provided for the article by Kuzu et al. [(2016), Acta Cryst. D72, 1137-1148].

After the online publication of the article by Kuzu et al. (2016), Drs Sjoer de Vries and Alexandre Bonvin noticed an apparent anomaly in the i.r.m.s.d. values in Table 6. Indeed, there were erroneous and duplicate entries in this table. (i) The values in the old Table 6 were computed using a definition which is different from that used in CAPRI. In the paper, the superposition was performed for the entire protein, and the r.m.s.d. was then computed only for the interface residues. We have revised Table 6 by recalculating i.r.m.s.d.

Table 6

Performance of our method on the HADDOCK-EM set (van Zundert et al., 2015) where cases were selected from the ZDOCK benchmark set.

Models were compared with the PDB structures and the results are presented with i.r.m.s.d. values. The first column gives the PDB code of the complex. The second column indicates the difficulty level assigned as in the benchmark data set. The third column lists the HADDOCK-EM results using unbound structures (listed in the benchmark). The fourth column gives the PRISM-EM results using the same set of unbound structures. Note that for equal comparison, columns 3 and 4 should be used. The fifth column lists the i.r.m.s.d. values of PRISM$E M$ using the alternative structures of the same proteins available in the PDB. The sixth column lists the i.r.m.s.d. values when the individual proteins are from the bound complex structures. A dash indicates a case where PRISM-EM could not find a model. Asterisks indicate use of the self-template interfaces of bound structures.

\begin{tabular}{|c|c|c|c|c|c|}
\hline \multirow[b]{3}{*}{ Complex } & \multirow[b]{3}{*}{ Difficulty } & \multicolumn{4}{|l|}{ I.r.m.s.d. (§) } \\
\hline & & \multirow{2}{*}{$\begin{array}{l}\text { HADDOCK-EM (itw) } \\
\text { Query (unbound) } \\
\text { proteins }\end{array}$} & \multicolumn{3}{|l|}{ PRISM-EM } \\
\hline & & & $\begin{array}{l}\text { Query (unbound) } \\
\text { proteins }\end{array}$ & $\begin{array}{l}\text { Query proteins and } \\
\text { their alternatives }\end{array}$ & $\begin{array}{l}\text { Bound } \\
\text { proteins }\end{array}$ \\
\hline 1avx & Easy & 0.67 & 1.72 & 1.01 & 0.51 \\
\hline 2oul & Easy & 0.63 & 0.83 & 0.83 & 0.55 \\
\hline 1ay7 & Easy & 0.66 & 1.25 & 1.19 & 0.75 \\
\hline $4 \mathrm{cpa}$ & Easy & 0.94 & - & - & - \\
\hline 1ahw & Easy & 0.91 & 1.23 & 1.23 & $0.47^{*}$ \\
\hline 7cei & Easy & 0.78 & 0.90 & 0.90 & 0.53 \\
\hline $2 o o b$ & Easy & 0.97 & 7.43 & 7.43 & $0.73^{*}$ \\
\hline $2 \mathrm{fd} 6$ & Easy & 1.13 & 1.43 & 0.94 & 0.45 \\
\hline $1 \mathrm{ak} 4$ & Easy & 1.22 & 1.40 & 1.40 & 1.01 \\
\hline $1 b 6 c$ & Easy & 1.88 & 2.22 & 2.22 & 0.74 \\
\hline $1 \operatorname{bg} x$ & Medium & 4.85 & 7.18* & 7.18* & $0.53^{*}$ \\
\hline $1 \mathrm{r} 6 \mathrm{q}$ & Medium & 1.26 & 9.38 & 0.95 & 0.72 \\
\hline $1 \mathrm{~m} 10$ & Medium & 2.82 & 2.59 & 0.93 & 0.47 \\
\hline $1 \mathrm{acb}$ & Medium & 2.43 & 9.24 & 0.68 & $0.59 *$ \\
\hline $1 \mathrm{jk} 9$ & Hard & 2.32 & 3.11 & 3.11 & 0.49 \\
\hline $1 \mathrm{bkd}$ & Hard & 3.62 & 4.61 & 0.92 & 0.49 \\
\hline 1jmo & Hard & 4.23 & 15.62 & 15.62 & $3.43^{*}$ \\
\hline
\end{tabular}




\section{addenda and errata}

values as described in CAPRI evaluations (Mendez et al., 2003) using the script irsmd.py (Viswanath et al., 2013), considering only the interface backbone atoms. (ii) The results for alternative conformations were compared with the rigidbody docking of $H A D D O C K-E M$ (it0) in the old table. We now compare these results with their explicit solvent docking results (itw), which are their best generated complexes among the top 400 solutions, with our results for both unbound and alternative structures. We have revised Table 6 accordingly. Using only unbound structures, $H A D D O C K$-EM (itw) models all 17 cases, where 15 of them have i.r.m.s.d. values less than $4 \AA$ A. PRISM-EM models ten of the 17 cases using unbound structures, where only one model has a better i.r.m.s.d. (1m10) than $H A D D O C K-E M$ models. Therefore, using unbound structures, HADDOCK-EM outperforms PRISM-EM (comparison of columns 3 and 4). When PRISM-EM considers alternative structures, the following cases additionally become better than HADDOCK-EM: 2fd6 (easy), 1r6q (medium), $1 \mathrm{acb}$ (medium) and 1bkd (hard). The supplementary tables have been revised to provide the alternative structures which have been used in the calculations. All of the models of Table 6 are also provided in the Supporting Information.

The authors apologize for any inconvenience that this has caused.

\section{References}

Kuzu, G., Keskin, O., Nussinov, R. \& Gursoy, A. (2016). Acta Cryst. D72, 1137-1148.

Mendez, R., Leplae, R., De Maria, L. \& Wodak, S. J. (2003). Proteins, 52, 51-67.

Viswanath, S., Ravikant, D. V. \& Elber, R. (2013). Proteins, 81, 592606.

Zundert, G. C. P. van, Melquiond, A. S. J. \& Bonvin, A. M. J. J. (2015). Structure, 23, 949-960. 